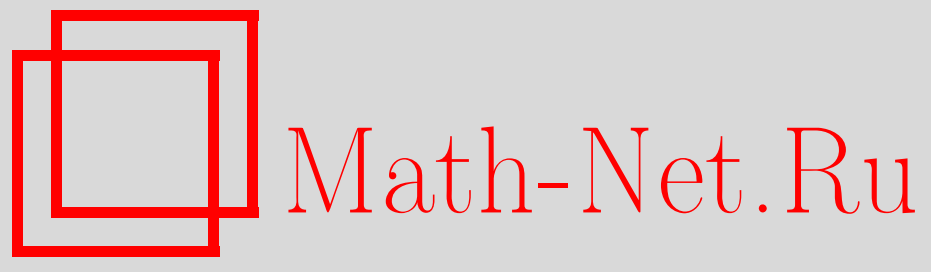

А. М. Головина, Резольвенты операторов с разбегающимися возмущениями, Матем. заметки, 2012, том 91, выпуск $3,464-466$

DOI: https://doi.org/10.4213/mzm9318

Использование Общероссийского математического портала Math-Net.Ru подразумевает, что вы прочитали и согласны с пользовательским соглашением http://www . mathnet.ru/rus/agreement

Параметры загрузки:

IP: 3.95 .254 .165

26 апреля 2023 г., 13:33:33

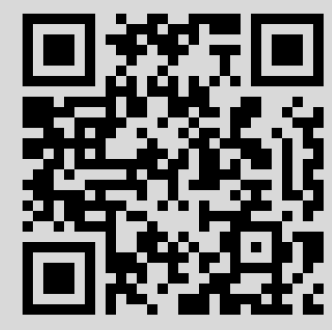




\section{Резольвенты операторов с разбегающимися возмущениями}

\section{А. М. Головина}

Большое количество работ посвящено изучению операторов с разбегающимися возмущениями (см., например, [1]-[4]). В основном внимание уделялось исследованию асимптотического поведения собственных значений и собственных функций оператора Шрёдингера с возмущениями, описываемыми вещественными потенциалами. В работе [1] рассматривался оператор Шрёдингера с двумя вещественными потенциалами из класса Рольника (Rollnik class) в пространстве $\mathbb{R}^{3}$. Предполагалось, что области, где локализованы данные потенциалы, находятся на большом расстоянии друг от друга. Была доказана сходимость резольвенты возмущенного оператора к резольвенте невозмущенного. В [2] исследовался аналогичный оператор, где потенциалы предполагались вещественными и убывающими. На основе возмущенного оператора был построен матричный оператор с разбегающимися возмущениями, а также доказана сходимость резольвенты унитарного преобразования данного матричного оператора. Отметим, что унитарное преобразование строилось определенным образом и зависело от расстояния между разбегающимися потенциалами. В [3] вновь рассматривался оператор Шрёдингера в пространстве $\mathbb{R}^{3}$ с тремя вещественными потенциалами. На потенциалы накладывались следующие условия: оператор $V\left(\mathrm{I}+\mathscr{H}_{0}\right)^{-1}$ компактный, где $\mathscr{H}_{0}$ - невозмущенный оператор, а $V$ - потенциал, и оператор

$$
D(\lambda) V\left(\mathrm{I}+\mathscr{H}_{0}\right)^{-1} D(\lambda)^{-1}, \quad \lambda \in \mathbb{R}_{+}^{3},
$$

является аналитическим продолжением ограниченного оператора $V\left(\mathrm{I}+\mathscr{H}_{0}\right)^{-1}$ в пространстве $L_{2}\left(\mathbb{R}^{3}\right)$ и действует из $\mathbb{R}_{+}^{3}$ в сектор

$$
S_{\phi}=\left\{\lambda \in \mathbb{C}^{3}\left|\operatorname{Re} \lambda_{l}>0,\right| \arg \lambda_{l} \mid<\phi, l=1,2,3\right\},
$$

где $\phi>0$,

$$
(D(\lambda) f)(y)=\prod_{l=1}^{3} \lambda_{l}^{1 / 2} f(\lambda y), \quad \lambda y=\left(\lambda_{1} y_{1}, \lambda_{2} y_{2}, \lambda_{3} y_{3}\right) .
$$

Доказана сходимость резольвенты возмущенного оператора к резольвенте невозмущенного оператора в смысле сильной резольвентной сходимости. Приведено разложение резольвенты возмущенного оператора в ряд Неймана, сходящийся в смысле сильной резольвентной сходимости. В [4] возмущающими операторами были произвольные абстрактные локализованные операторы, локализованность которых заключалась в том, что каждый из них был задан на определенной ограниченной области. Были доказаны теоремы сходимости и построены первые члены асимптотических разложений собственных значений и соответствующих им собственных функций.

В данной работе рассматривается матричный оператор общего вида с конечным числом локализованных возмущений. Невозмущенным является дифференциальный оператор высокого порядка, не обязательно симметричный. Главное условие - замкнутость невозмущенного оператора. Строго он вводится следующим образом.

Пусть $x=\left(x_{1}, \ldots, x_{d}\right)$ - декартовы координаты в $\mathbb{R}^{d}, d \geqslant 1, \Gamma$ - произвольная периодическая решетка в $\mathbb{R}^{d}$ с элементарной ячейкой $\square$. Определим в пространстве $L_{2}\left(\mathbb{R}^{d} ; \mathbb{C}^{n}\right)$ невозмущенный оператор

$$
\mathscr{H}_{0}:=(-1)^{m} \sum_{\substack{\beta, \gamma \in \mathbf{Z}_{+}^{d} \\|\beta|=|\gamma|=m}} \frac{\partial^{\beta}}{\partial x^{\beta}} a_{\beta \gamma}(x) \frac{\partial^{\gamma}}{\partial x^{\gamma}}+\sum_{\substack{\beta \in \mathbf{Z}_{+}^{d} \\|\beta| \leqslant 2 m-1}} b_{\beta}(x) \frac{\partial^{\beta}}{\partial x^{\beta}},
$$

Работа выполнена при финансовой поддержке Российского фонда фундаментальных исследований (грант № 10-01-00118), программы Президента России поддержки молодых ученых (грант № МД-453.2010.1) и ФЦП (грант № 02.740.110612). 
с областью определения $W_{2}^{2 m}\left(\mathbb{R}^{d} ; \mathbb{C}^{n}\right)$, где $m \in \mathbb{N}, a_{\beta \gamma} \in C^{m}\left(\mathbb{R}^{d}\right), b_{\beta} \in C^{m-1}\left(\mathbb{R}^{d}\right)$ - матричнозначные функции, периодические относительно решетки Г. Будем считать, что выполнено условие эллиптичности:

$$
\nu \sum_{\substack{\beta \in Z_{+}^{d} \\|\beta|=m}}\left|\xi_{\beta}\right|^{2} \leqslant\left|\sum_{\substack{\beta, \gamma \in Z_{+}^{d} \\|\beta|=|\gamma|=m}}\left(a_{\beta \gamma} \xi_{\beta}, \xi_{\gamma}\right)_{\mathbb{C}^{d}}\right|, \quad \xi_{\beta} \in \mathbb{C}^{d},
$$

где $\nu$ - некоторая константа, не зависящая от $x$ и $\xi_{\beta}$.

Для определения возмущающих операторов введем в рассмотрение произвольные абстрактные локализованные операторы $\mathscr{L}_{i}^{0}: W_{2}^{2 m}\left(\mathbb{R}^{d} ; \mathbb{C}^{n}\right) \rightarrow L_{2}\left(\mathbb{R}^{d} ; \mathbb{C}^{n}\right), i=1, \ldots, k$, ограниченные как операторы из $W_{2}^{2 m}\left(\mathbb{R}^{d} ; \mathbb{C}^{n}\right)$ в $L_{2}\left(\mathbb{R}^{d} ; \mathbb{C}^{n}\right)$. Локализованность возмущающих операторов заключается в специально выбранных весовых функциях $\varsigma_{i}=\varsigma_{i}(r), \eta_{i}=\eta_{i}(r) \in$ $C^{2 m}\left(\mathbb{R}_{+}\right), i=1, \ldots, k$, которые неотрицательны, в окрестности нуля равные единице и удовлетворяют следующим условиям:

(A1) существует функция $а \in C^{2 m-1}[0,+\infty)$ такая, что справедливы оченки: $\left|\varsigma_{i}(r)\right| \leqslant$ $C e^{-\int_{0}^{r} a(t) d t}, i=1, \ldots, k$, где $C$ - некоторая константа;

(A2) функция а и все ее производные до порядка $(2 m-1)$ ограничены равномерно в $\mathbb{R}^{d}$, а также выполнено равенство $\int_{0}^{+\infty} a(t) d t=+\infty$;

(A3) функция $a \equiv 0$, а функции $\eta_{i} \equiv 1$ при $r \in\left[0, r_{0}\right]$, где $r_{0}>0$ - некоторое фиксированное число, функиии $\eta_{i}$ и все их производные до порядка $2 m$ убывают на бесконечности.

Под разбегающимися возмущениями оператора $\mathscr{H}_{0}$ будем понимать возмущения вида

$$
\sum_{i=1}^{k} \mathscr{S}\left(-X_{i}\right) \varsigma_{i}(|x|) \mathscr{L}_{i}^{0} \eta_{i}(|x|) \mathscr{S}\left(X_{i}\right),
$$

где $\mathscr{S}\left(X_{i}\right)$ - оператор сдвига, действующий по правилу: $\left(\mathscr{S}\left(X_{i}\right) u\right)(\cdot):=u\left(\cdot-X_{i}\right), X_{i} \in \mathbb{R}^{d}-$ дискретные параметры, стремящиеся к бесконечности. Заметим, что любые два значения $X_{i}$ можно перевести друг в друга с помощью конечного числа сдвигов вдоль решетки Г и $\left|X_{i}-X_{j}\right| \underset{i \neq j}{\longrightarrow}+\infty$. Описанное поведение параметров $X_{i}$ далее будем кратко записывать в виде $X \rightarrow \infty$, где $X=\left(X_{1}, \ldots, X_{k}\right)$.

Под возмущенным оператором в пространстве $L_{2}\left(\mathbb{R}^{d} ; \mathbb{C}^{n}\right)$ будем понимать оператор

$$
\mathscr{H}_{X}:=\mathscr{H}_{0}+\sum_{i=1}^{k} \mathscr{S}\left(-X_{i}\right) \varsigma_{i} \mathscr{L}_{i}^{0} \eta_{i} \mathscr{S}\left(X_{i}\right)
$$

с областью определения $W_{2}^{2 m}\left(\mathbb{R}^{d} ; \mathbb{C}^{n}\right)$. Самым простым примером возмущенного оператора является одномерный периодический дифференциальный оператор второго порядка с двойной потенциальной ямой. В данном случае $m=d=n=1, \mathscr{L}_{i}^{0}=\mathrm{V}_{i}$, где $\mathrm{V}_{i}-$ некоторые ограниченные потенциалы, $i=1,2$. Возмущенный оператор $\mathscr{H}_{X}$ в этом случае рассматривается в пространстве $L_{2}(\mathbb{R})$ с областью определения $W_{2}^{2}(\mathbb{R})$ :

$$
\begin{gathered}
\mathscr{H}_{\ell}=\mathscr{H}_{0}+\varsigma_{1}(|\cdot+\ell|) \mathrm{V}_{1}(\cdot+\ell) \eta_{1}(|\cdot+\ell|)+\varsigma_{2}(|\cdot-\ell|) \mathrm{V}_{2}(\cdot-\ell) \eta_{2}(|\cdot-\ell|), \\
\mathscr{H}_{0}=-\frac{d}{d x} p \frac{d}{d x}+q,
\end{gathered}
$$

где $p \in C^{1}(\mathbb{R}), q \in C(\mathbb{R})$ - 1-периодические функции, $\ell$ - большой положительный параметр.

Для формулировки основного результата введем дополнительные обозначения. Пусть $\mathscr{H}_{i}:=\mathscr{H}_{0}+\varsigma_{i} \mathscr{L}_{i}^{0} \eta_{i}-$ операторы в пространстве $L_{2}\left(\mathbb{R}^{d} ; \mathbb{C}^{n}\right)$ с областями определения $W_{2}^{2 m}\left(\mathbb{R}^{d} ; \mathbb{C}^{n}\right)$. Будем предполагать, что

(A4) операторь $\mathscr{H}_{i}$ замкнуты. 
Через $\sigma(\cdot)$ обозначим спектр оператора, I - тождественный оператор, а символом $\|\cdot\|_{Y_{1} \rightarrow Y_{2}}$ будем обозначать норму линейного оператора, действующего из пространства $Y_{1}$ в пространство $Y_{2}$.

Основной результат сформулируем в виде следующей теоремы.

Теорема 1. Для достаточно больиих $X$ оператор $H_{X}$ замкнут. Для любого $\lambda \in \mathbb{C} \backslash$ $\bigcup_{i=0}^{k} \sigma\left(\mathscr{H}_{i}\right)$ и достаточно больших $X$ верно представление

$$
\begin{gathered}
\left(\mathscr{H}_{X}-\lambda\right)^{-1}:=\left[\sum_{i=1}^{k} \mathscr{S}\left(-X_{i}\right)\left(H_{i}-\lambda\right)^{-1} \mathscr{S}\left(X_{i}\right)-(k-1)\left(\mathscr{H}_{0}-\lambda\right)^{-1}\right]\left(\mathrm{I}+\mathscr{P}_{X}\right)^{-1}, \\
\mathscr{P}_{X}:=\sum_{\substack{i, j=1 \\
i \neq j}}^{k} \mathscr{S}\left(-X_{i}\right) \varsigma_{i} \mathscr{L}_{i}^{0} \eta_{i} \mathscr{S}\left(X_{i}\right)\left[\mathscr{S}\left(-X_{j}\right)\left(\mathscr{H}_{j}-\lambda\right)^{-1} \mathscr{S}\left(X_{j}\right)-\left(\mathscr{H}_{0}-\lambda\right)^{-1}\right],
\end{gathered}
$$

где $\left\|\mathscr{P}_{X}\right\|_{L_{2}\left(\mathbb{R}^{d} ; \mathbb{C}^{n}\right) \rightarrow L_{2}\left(\mathbb{R}^{d} ; \mathbb{C}^{n}\right)} \rightarrow 0$ nрu $X \rightarrow \infty$.

Для приведенного выше оператора (1) резольвента имеет вид

$$
\begin{aligned}
\left(\mathscr{H}_{\ell}-\lambda\right)^{-1}=\left(\mathscr{S}(-\ell)\left(\mathscr{H}_{0}+\varsigma_{1} \mathrm{~V}_{1} \eta_{1}-\lambda\right)^{-1} \mathscr{S}(\ell)\right. & \left.+\mathscr{S}(\ell)\left(\mathscr{H}_{0}+\varsigma_{2} \mathrm{~V}_{2} \eta_{2}-\lambda\right)^{-1} \mathscr{S}(-\ell)-\left(\mathscr{H}_{0}-\lambda\right)^{-1}\right)\left(\mathrm{I}+\mathscr{P}_{\ell}\right)^{-1} \\
& \quad \mathscr{P}_{\ell}=\mathscr{S}(-\ell) \varsigma_{1} \mathrm{~V}_{1} \eta_{1} \mathscr{S}(\ell)\left(\mathscr{S}(\ell)\left(\mathscr{H}_{0}+\varsigma_{2} \mathrm{~V}_{2} \eta_{2}-\lambda\right)^{-1} \mathscr{S}(-\ell)-\left(\mathscr{H}_{0}-\lambda\right)^{-1}\right) \\
& +\mathscr{S}(\ell) \varsigma_{2} \mathrm{~V}_{2} \eta_{2} \mathscr{S}(-\ell)\left(\mathscr{S}(-\ell)\left(\mathscr{H}_{0}+\varsigma_{1} \mathrm{~V}_{1} \eta_{1}-\lambda\right)^{-1} \mathscr{S}(\ell)-\left(\mathscr{H}_{0}-\lambda\right)^{-1}\right) .
\end{aligned}
$$

Отметим, что класс невозмущенных и возмущающих операторов довольно широк. Например, в качестве невозмущенного оператора могут быть рассмотрены следующие операторы: дифференциальный оператор второго порядка, матричный и магнитный операторы Шрёдингера, оператор теории упругости, оператор Паули, бигармонический оператор, а также оператор, описывающий $\delta$-взаимодействие, который с помощью некоторого преобразования, описанного в работе [4], можно привести к дифференциальному оператору второго порядка. Возмущающими операторами могут быть дифференциальный оператор, интегральный оператор, конечномерный оператор, псевдодифференциальный оператор с определенным условием на символ оператора. При определенном соотношении между размерностью пространства и порядком невозмущенного оператора в качестве возмущений можно взять кулоновские потенциалы.

Автор выражает благодарность Д. И. Борисову за постановку задачи и консультирование в процессе написания работы.

\section{СПИСОК ЦИТИРОВАННОЙ ЛИТЕРАТУРЫ}

[1] V. Kostrykin, R. Schrader, Rev. Math. Phys., 6:5 (1994), 833-853. [2] E. B. Davies, Spectral Theory and Differential Operators, Cambridge Stud. Adv. Math., 42, Cambridge Univ. Press, Cambridge, 1995. [3] P. Aventini, R. Seiler, Comm. Math. Phys., 41:2 (1975), 119-134. [4] D. Borisov, Math. Phys. Anal. Geom., 10:2 (2007), 155-196.

\section{А. М. Головина}

Поступило

Башкирский государственный педагогический

20.06.2011

университет им. М. Акмуллы, г. Уфа

E-mail: nastya_gm@mail.ru 